\title{
A Modification in the Shape of the Skin Island of Fasciocautaneous Flaps in the Distal Extremities
}

\author{
AHMED ABDEL-SALAM HAFIZ, M.D.; AHMED NAWAR, M.D. and AHMED MAHER SAMY, M.D. \\ The Department of General Surgery, Plastic Surgery Unit, Faculty of Medicine, Cairo University
}

\begin{abstract}
Background: Distally based (reversed flow) fasciocautaneous flaps are used for coverage of distal extremity soft tissue defects. However, there is a high incidence of venous congestion that may lead to partial or total flap loss. Various modifications for these flap design have been made aiming to improve flap survival.
\end{abstract}

Aim of Work: In this paper, a tongue like extension from the skin island was done aiming to improve its versatility and decrease complication rate especially venous congestion.

Patients and Methods: Eight patients (five males and three females), average age thirty years old. Most cases where post traumatic, one case necrotizing fasciitis of heel, one case with squamous cell cancer of dorsal hand skin. Five patients underwent reversed sural flap and three patients underwent radial forearm flap.

Results: All cases had viable flaps with good wound healing. However female patients complained of the scarring later on. One case of reversed sural flap had minimal distal congestion that improved later on.

Conclusion: Advantage of this modification is that it acts to stabilize the critical vascular pattern of the pedicle during its axial rotation minimizing the post insetting vascular problem. It improves the venous drainage through the subdermal and subcutaneous venous plexuses and limits the excess kinking of the fascial pedicle. This tongue of tissue can also serve as an extra tissue to be used for coverage in some cases. It also has a superior cosmetic appearance when compared to skin grafting of the pedicle. The use of this modified technique can be extended to any island flap that has to pass through a tight tunnel to reach the defect.

Key Words: Distal extremities - Venous congestion -Fasciocautaneous flaps - Adipofascial pedicle - Skin grafting - Tongue like modification - Skin Island.

\section{INTRODUCTION}

Coverage of soft tissue defects in the distal extremities is challenging. Local regional flaps selection depends on the size, location and function of the site requiring reconstruction. However, some cases may require free flaps. Distal extremities defects usually require reverse flow fasciocutaneous flaps [1]. These flaps are usually designed in the form of Skin Island with adipofascial pedicle providing a wide arc of rotation, easy and quick elevation and acceptable donor site morbidity. However, venous congestion may occur especially if tunneled subcutaneously due to postoperative edema in a tough inelastic skin in the extremities causing pressure to the vascular pedicle in the subcutaneous tunnel which may lead to partial or total flap loss $[2,3]$. The overall incidence of venous congestion varies widely between $5 \%$ to $80 \%$. Accordingly several modifications had been made in order to decrease venous congestion [4-8]. However; most of the described modifications decrease the relative easy dissection of the flaps with some residual limitations and drawbacks [9].

In this paper a modification in the form of a tongue shaped extension from the skin island of fasciocautaneous flaps is described to cover defects in the distal extremities in order to improve flap versatility and decrease flap complications.

\section{PATIENTS AND METHODS}

Eight patients (5 males and 3 females) with small to medium sized defects of the distal extremities were operated upon in the period from September 2015 - September 2017 in kasr alaini hospital. Their age ranged from 16-50 years old (average age 33 years old). Three patients underwent radial forearm flap for coverage of dorsal hand defects (one post excision of squamous cell cancer and two post traumatic). Five patients had reversed sural flap (one with necrotizing fasciitis over tendoachillis tendon and four post traumatic heel and lower leg defects).

\section{Surgical technique:}

A modification was used in the shape of Skin Island in the form of a tongue shaped extension 
with its tip pointing to the pivot point. The length of this tongue shaped extension varies as it extends all over the pedicle length till the pivot point. The width of the strip of skin ranges from $2-4 \mathrm{~cm}$ allowing primary closure of its donor site. The flap is transposed through an open incision instead of being tunneled to reach the recipient defect. The open incision is closed by this tongue shaped extension overlying the pedicle. The defect of the donor site is covered with split thickness skin graft.

\section{RESULTS}

In this paper, eight patients underwent the modified island flap for soft tissue reconstruction in 3 hand defects and 5 lower extremity defects. All the flaps survived and healed well with no evidence of venous congestion except one case that had minimal distal congestion of the reversed sural flap due to a hematoma that was evacuated and the flap distal flap margin improved (Figs. 3,4). Neither significant short term or long term post-operative complications were noted. (Table 1).

The case samples are depicted in Figs. (1-5):

Case (1): Male patient, 27 years old with necrotizing fasciitis at tendoachilis region. Debridement was done followed by coverage with the modified skin island of reversed sural flap. Initial congestion of a few millimeters of the distal flap due to a hematoma that was rapidly evacuated with improvement of the circulation and complete flap survival (Figs. 1-3).

Case (2): Male patient, 45 years old with post traumatic degloving injury with exposed extensor tendons of the left hand. A radial forearm flap with the modified skin island was used for coverage. The flap completely survived including the tongue like extension crossing the wrist joint avoiding wrist joint contracture (Figs. 4,5).

Table (1): Showing summary of the cases.

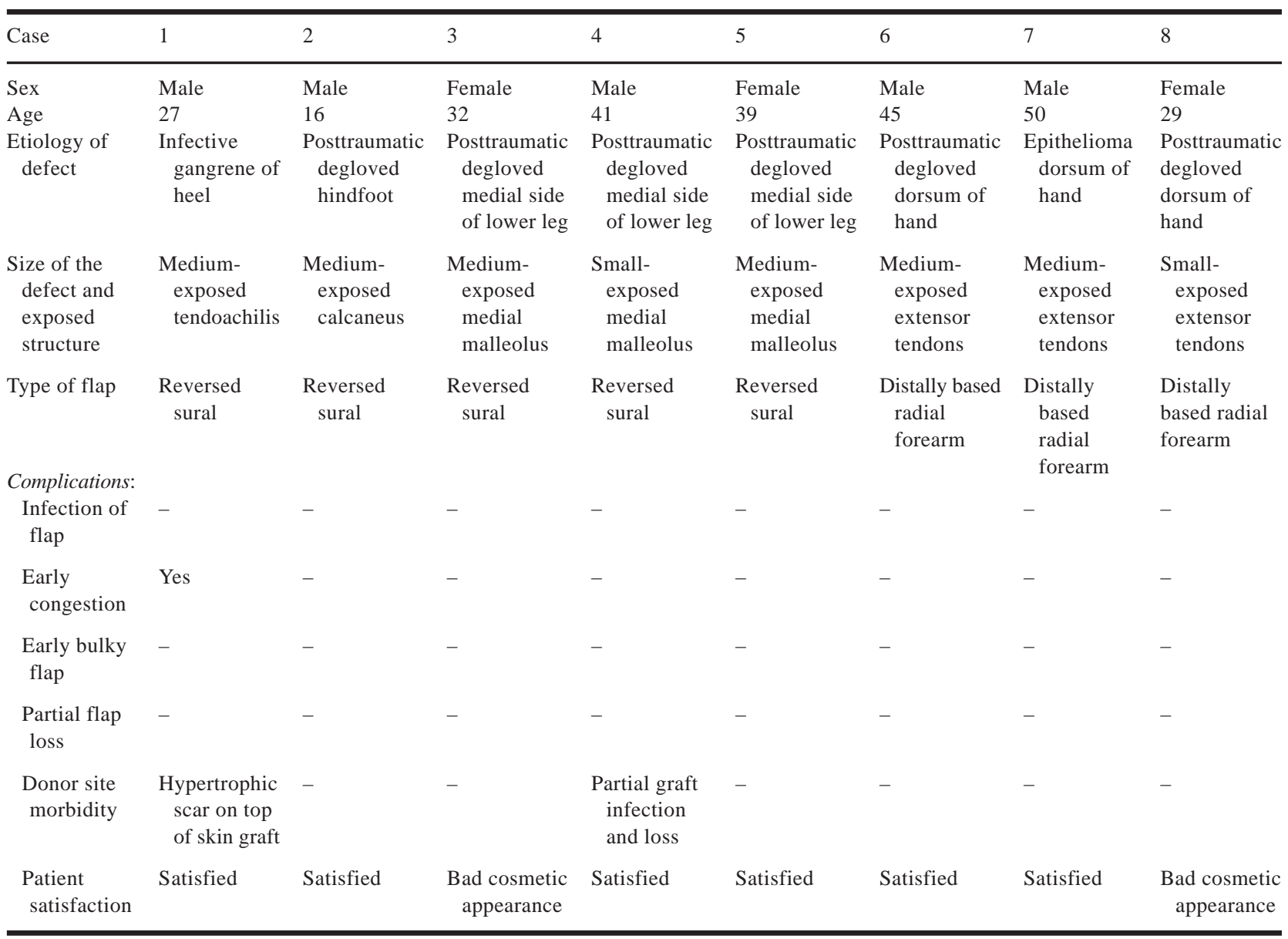


Fig. (1): Showing modification in the shape of the skin island of reversed sural flap for coverage of exposed tendoachillis tendon (Skin Island with a tongue shaped extension).

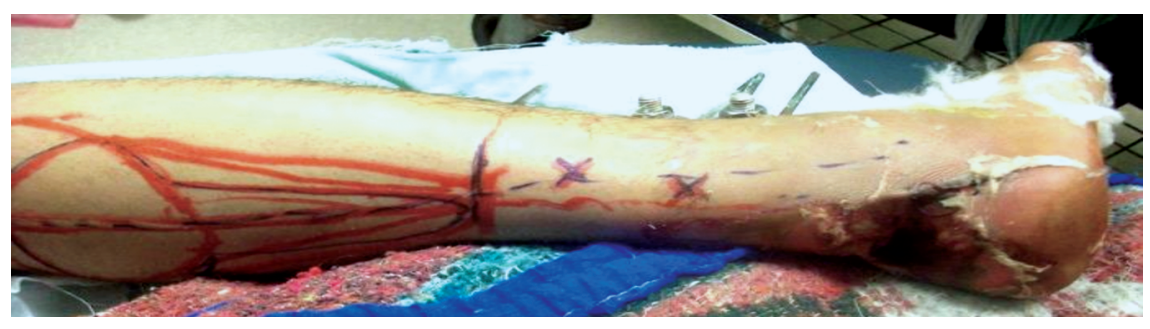

Fig. (2): Showing postoperative picture of same previous patient (note the tongue shaped extension of the skin island overlying the pedicle of the flap and the minimal distal congestion).

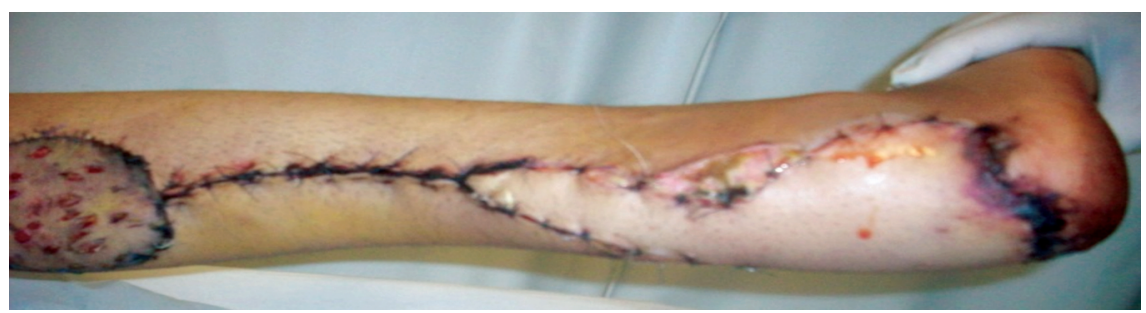

Fig. (3): Of same patient after six months showing complete flap survival.

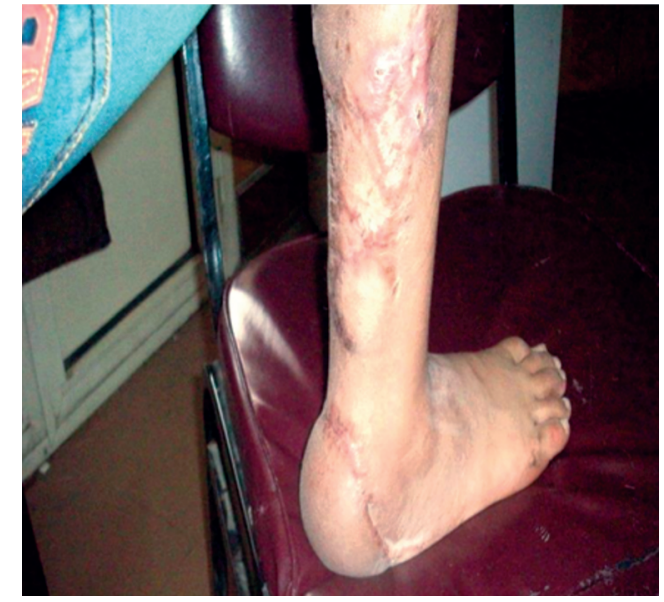

Fig. (4): Showing modification in the shape of the skin island of radial forearm flap for coverage of exposed extensor tendons of the hand (Skin Island with a tongue shaped extension).

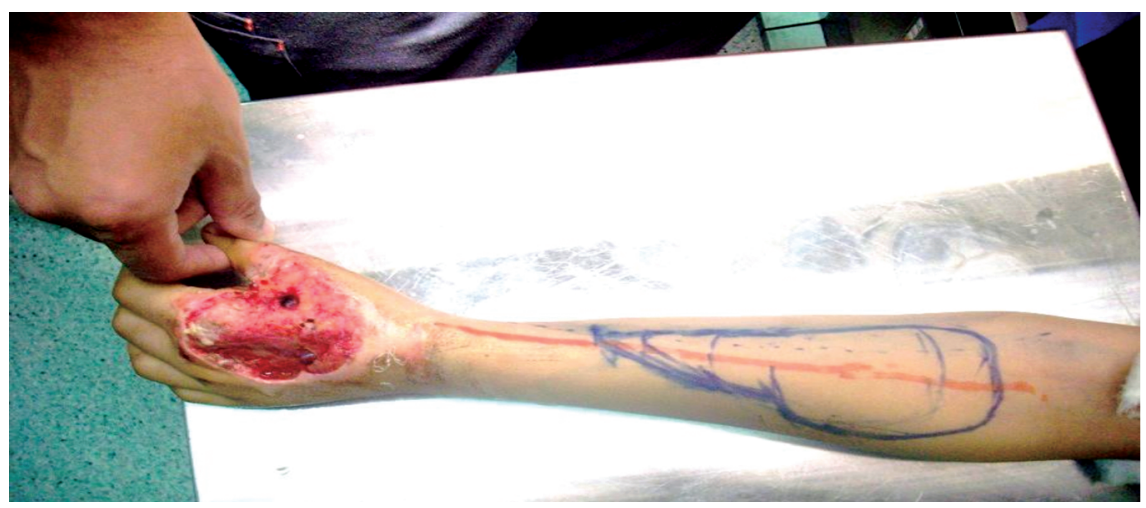

Fig. (5): Showing postoperative picture of same previous patient (note the tongue shaped extension of the skin island overlying the pedicle of the flap crossing the wrist).

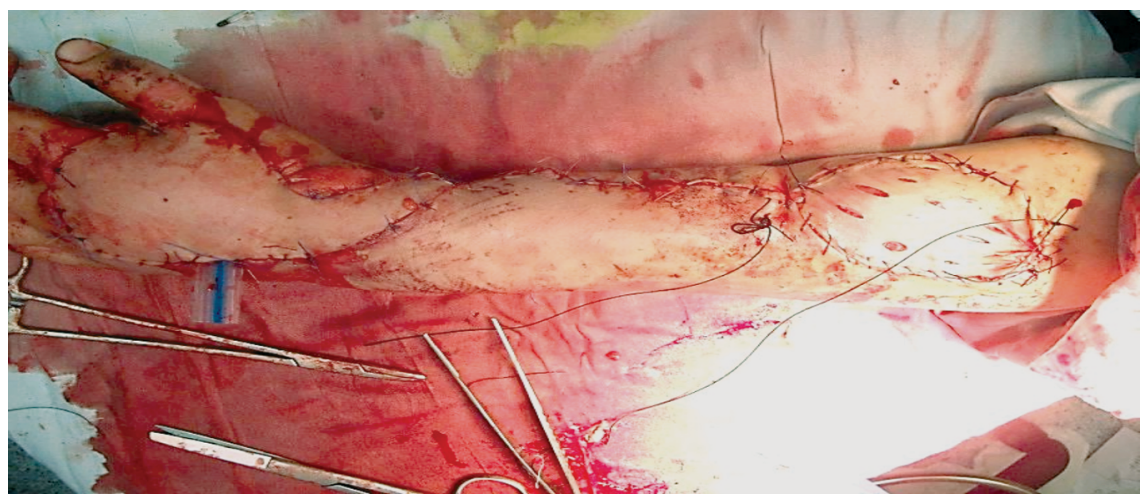




\section{DISCUSSION}

Soft tissue reconstruction of defects in the distal extremities is challenging. Loco-regional, distant and free flaps are the various options each with its various advantage, disadvantage, indications and contraindications. Island pedicled loco-regional flaps can be raised during one stage procedure, not requiring microvascular expertise. However, the main complication that may occur is venous congestion especially if the flap pedicle is tunneled subcutaneously. Although many authors [10,11] adopted incision of this skin tunnel and skin grafting of the adipofascial pedicle, some cases still developed flap venous congestion, a bulky pedicle with unacceptable cosmetic appearance, graft loss or even flap loss due to infection and poor graft take by the pedicle fatty tissue. Various modifications have been described to improve the survival of these flaps [4-8]. In this study we suggested a modification in the shape of the tongue like extension from the skin island overlying the adipofascial pedicle with its apex directed towards the pivot point. Thus aiming to improve flap survival by decreasing venous congestion and infection with a better cosmetic appearance.

The reversed sural flap could be used for defects in the distal third of the leg and hind foot. However, venous congestion may occur leading to partial or total flap necrosis [7,10-12]. Modifications have been described to decrease this venous congestion. Elshazly and Yassin [6] used a broad inferolateral adipofascial pedicle that includes extensive veins superior and posterior to the lateral malleolus.

Al-Qattan [7] modified the flap by including a part of the gastrocnemius muscle in the pedicle to help in venous drainage. Maffi et al. [12] used the interpolation flap design. However second stage is required for flap separation. Dragu et al. [5] included the cutis and subcutis with the pedicle to prevent undue tension or tourniquet effect on the pedicle during its rotation. They also advised for a venous anastomosis of the proximal end of the sural flap vein with a recipient vein if flap congestion is present intraoperatively. Buluc et al described rapid soft tissue expansion of the tunnel to prevent flap compression [8].

The distally based radial forearm flap has similar concept to the reversed sural flap in the distal upper extremity defects $[\mathbf{1 , 3 , 1 3 , 1 4 ]}$. However, there is the main disadvantage of unacceptable cosmetic appearance especially in female patients. The standard pedicle dissection may produce superficial epidermolysis of the skin overlying the pedicle with subsequent delayed wound healing or even wound dehiscence due to the excess subdermal thinning to include the loose subcautaneous tissue with the cephalic vein in the pedicle. Also postsurgical edema may compress the pedicle in the tight tunnel at the wrist joint. This modification in the skin island avoided the need of subdermal thinning and flap tunneling leading to good wound healing and good viable flaps respectively.

\section{Conclusion:}

The tight skin around the defect in the distal extremities either caused by inflammatory edema or fibrotic induration as an influence of trauma made tunneling of the flap hazardous which might have a bad impact on the vascularity of the fasciocautaneous flaps. We usually used to lay open the skin bridge and apply a graft over the pedicle. Inadequate graft take might expose the flap pedicle to the risk of infection that could endanger the flap itself. The later modification of the skin island in the form of a tongue shaped extension towards the pivot point in dimensions that allow comfortable adaptation in the corresponding incision of the tough skin nearby the defect avoided the risk of applying a graft over the pedicle besides it added no further morbidity to the donor site as it was almost exclusively closed primarily. Another potential advantage of this modification is that it acts to stabilize the critical vascular pattern of the pedicle during its axial rotation minimizing the post insetting vascular problem. It improves the venous drainage through the subdermal and subcutaneous venous plexuses and limits the excess kinking of the fascial pedicle. This tongue of tissue can also serve as an extra tissue to be used for coverage in some cases. It also has a superior cosmetic appearance when compared to skin grafting of the pedicle. The use of this modified technique can be extended to any island flap that has to pass through a tight tunnel to reach the defect.

\section{REFERENCES}

1- Li J-H., Xing X., Ouyang T-X., Li P. and Xu J.: An innovation in the subcutaneous island pedicle flap for cutaneous reconstruction. J. Plast. Reconstr. And Aesthetic Surgery, 59: 174-180, 2006.

2- Kneser U., Bach A.D., Polykandriotis E., Kopp J. and Horsh R.E: Delayed reverse sural flap for staged reconstruction of the foot and lower leg. Plast. Reconstr. Surg., 116 (7): 1910-17, 2005.

3- Sabapathy S.R. and Bhardwaj P.: Skin cover in hand injuries. In mini-symposium: Hand trauma. Current Orthopaedic., 22: 1-8, 2008.

4- Li Y-F. and Lao J.: A simple design to improve venous drainage in retrograde island flaps: Surgical technique. J. Plast. Reconstr. and Aesthet. Surg., 63: e211-e212, 2010. 
5- Dragu A., Bach A.D, Kneser U. and Horch R.E.: Two easy and simple modifications when using a distally based sural flap to reduce the risk of venous congestion. Plast. Reconstr. Surg. Let. August, 122 (2): 683-84, 2008.

6- El-Shazly M. and Yassin O.: Increasing the success rate of the reverse-flow fasciocutaneous island sural flap.A clinical experience in 26 cases. Ann. Plast. Surg., 57: 653-657, 2006.

7- Al-Qattan M.M.: A modified technique for harvesting the reverse sural artery flap from the upper part of the leg: Inclusion of a gastrocnemius muscle"cuff" around the sural pedicle. Ann. Plast. Surg., 47: 269, 2001.

8- Buluc L., Tosun B., Sen C. and Sarlak A.Y.: A modified technique for transposition of the reverse sural artery flap. Plast. Reconstr. Surg., 117: 2488, 2006.

9- Loonen M.P.J., Moshe K. and Schuurman A.H.: Correspondence letter. Plast. Reconstr. Surg. August, p 684 2008.
10- Akhtar S. and Hameed A.: Versatility of the sural fasciocautaneous flap in the coverage of the lower third leg and hind foot defects. J. of Plast., Reconstr. And Aesthet. Surg., 59: 839-845, 2006.

11- Almeida M.F., da Costa P.R. and Okawa R.Y.: Reverse flow island sural flap. Plast. Reconstr. Surg., 109: 583, 2002.

12- Maffi T.R., Knoetgen III J., Turner N.S. and Moran S.L.: Enhanced survival using the distally based sural artery interpolation flap. Ann. Plast. Surg., 54: 302-305, 2005.

13- Soucacos P.N., Zoubos A.B., Korompilias A.V. and Vekris M.D: Versatility of the island forearm flap in the management of extensive skin defects of the hand. Injury, Int. J. Care Injured, 39s: S49-S56, 2008.

14- Mathes Stephen J. and Nahai Foad: Radial forearm flap, Reconstructive surgery, principles, anatomy and technique, Volume 2, p.775-802, 1997. 